\title{
Young Stellar Variability of GM Cephei by Circumstellar Dust Clumps
}

\author{
Po-Chieh Huang ${ }^{1 *}$, Wen-Ping Chen ${ }^{1}$, Chia-Ling $\mathrm{Hu}^{2}$, Otabek Burkhonov ${ }^{3}$ \\ Shuhrat Ehgamberdiev ${ }^{3}$, Jinzhong Liu ${ }^{4}$, Hiroyuki Naito ${ }^{5}$, Erika Pakstiene ${ }^{6}$ \\ Jan Kare Trandem Qvam ${ }^{7}$, Stefanie Rätz ${ }^{8}$, Evgeni Semkov ${ }^{9}$ \\ and YETI Collaboration
}

\author{
${ }^{1}$ Graduate Institute of Astronomy, National Central University, Taiwan \\ ${ }^{2}$ Taipei Astronomical Museum, Taiwan \\ ${ }^{3}$ Ulugh Beg Astronomical Institute, Uzbekistan \\ ${ }^{4}$ Nanshan 1m telescope of Xinjiang Astronomical Observatory, China \\ ${ }^{5}$ Nayoro Observatory, Japan \\ ${ }^{6}$ Institute of Theoretical Physics and Astronomy, Vilnius University, Lithuania \\ ${ }^{7}$ Mathematics and Natural Sciences, Department of Physics, University of Oslo, Norway \\ ${ }^{8}$ Freiburg Institute of Advanced Studies (FRIAS), University of Freiburg, Germany \\ ${ }^{9}$ Institute of Astronomy and National Astronomical Observatory, Bulgaria
}

\begin{abstract}
UX Orionis stars are a sub-type of Herbig Ae/be or T Tauri stars exhibiting sporadic extinction of stellar light due to circumstellar dust obscuration. GM Cep is such an UX Orionis star in the young ( $4 \mathrm{Myr}$ ) open cluster Trumpler 37 at $\sim 900 \mathrm{pc}$, showing a prominent infrared access, H-alpha emission, and flare activity. Our multi-color photometric monitoring from 2009 to 2016 showed (i) sporadic brightening on a time scale of days due to young stellar accretion, (ii) cyclic, but not strictly periodical, occultation events, each lasting for a couple months, with a probable recurrence time of about two years, (iii) normal dust reddening as the star became redder when dimmer, (iv) the unusual "blueing" phenomena near the brightness minima, during which the star appeared bluer when dimmer, and (v) a noticeable polarization, from 3 to 9 percent in $\mathrm{g}$, r', and i' -bands. The occultation events may be caused by dust clumps, signifying the density inhomogeneity in a young stellar disk from grain coagulation to planetesimal formation. The level of polarization was anticorrelated with the brightness in the bright state, when the dust clump backscattered stellar light. We discussed two potential hypotheses: orbiting dust clumps versus dust clumps along a spiral arm structure.
\end{abstract}

\section{Introduction}

Stars are formed in molecular cloud cores, whereas planets are condensed in young circumstellar disks. The dust grains grow in size since in the molecular cloud, and continue to become ever larger.

*Email:pochiehhuang1@gmail.com 
The initital step of planetesimal formation comes from coaguration of sub- $\mu$ m dust particles in the disks around pre-main-sequence stars (Natta et al. 2007). Two theories have been proposed: planetesimal accretion (Weidenschilling 2000) versus gravitational instability (Safronov 1972; Goldreich \& Ward 1973; Johansen 2007). In any case the process must start with density inhomogeneities in the disk. The estimated time scale for an optically thick disk is less than $\sim 10 \mathrm{Myr}$ (Mamajek et al. 2004; Briceo et al. 2007; Hillenbrand 2008), which corresponds to the pre-main-sequence phase from a classical T Tauri star to a weak-lined T Tauri star.

Herbst et al. (1994) studied dozens of young stars and categorized them into three major groups in accordance with the variation of the brightness and color behavior due to accretion of, or obscuration by, circumstellar material. Those with variation arising from abrupt accretion are called FUors, with FU Ori being the prototype, which brightened up to 6 mag in a few months, followed by a slow decline in years to decades, or EXors, named after EX Lupi, which brightened up to 5 mag and faded on about the same time scale. The third group, with variation caused by obscuration by circumstellar dust, are called UX Orionis (UXor) type variables, with typical periods known to range from $\sim 8.2 \mathrm{~d}$ (Bouvier et al. 2003) to $11.2 \mathrm{yr}$ (Grinin et al. 1998).

GM Cephei is an active T Tauri star in the young ( $<4$ Myr: Marshall et al. 1990; Patel et al. 1995; Sicilia-Aquilar et al. 2005) open cluster Trumpler 37. At a distance of $\sim 870 \mathrm{pc}$ (Contreras et al. 2002), GM Cep was first thought to be an abrupt variable, i.e., an EXor (Sicilia-Aquilar et al. 2008), but later Xiao et al. (2010) concluded that the variation in the brightness is caused by dip events superposed on quiescent states. GM Cep was also classified as a UXor by Chen et al. (2012), and by Semkov \& Peneva (2012). The existence of any periodicity of GM Cep remains unsettled: while Chen et al. (2012) suggested a possible recurrent time of dips in about 310 days, Semkov et al. (2015) later concluded otherwise.

\section{Repeated Occultation Events by Circumstellar Dust Clumps}

Here we present the BVR light curves of GM Cep, shown in Fig. 1a, from 2009 to March 2017, with supporting data from the Young Exoplanet Transit Initiative (YETI) collaboration (Neuhäuser et al. 2011), together with additional observations taken in Uzbekistan, Japan, China, Lithuania and Norway. Some major dips ( $>1 \mathrm{mag}$ ) in brightness can be seen, e.g., in mid-2009, mid-2010, end of 2011, beginning of 2014, mid-2015, and beginning of 2017, each lasting for a couple of months. Within the seven years of photometric monitoring, the dips seems repetitive, while not strictly periodical, with a recurrence time of about two years. Sporadic flare events were detected, each lasting for a couple of days. Such "cyclic but not exactly periodic" light curves have been seen among some Herbig Ae/Be stars (Herbst \& Shevchenko 1999).

It can be seen in Fig. 1b that the star becomes blue around brightness minima, opposite to the normal interstellar reddening. This "blueing effect", commonly found in UXors, is attributed to scattered light dominating the stellar light which is otherwise heavily obscured by the circumstellar dust.

The dynamical period analysis with a window size of 2000 days, by applying the Lomb-Scargle method (Lomb 1976; Scargle 1982), displays the possible existence of a quasi-period (see Fig. 1c), suggesting a broad range of periods from 500 to 900 days between 2009 to 2017. The strongest signal is near a period of about 710 days from 2012 to 2015. If the signal came from the orbiting dust clump, the estimated Keplerian orbital distance is about 2AU. It is puzzling how the dust clump survives the stellar tidal disruption. The alternative scenario is the presence of clumpy dust along a spiral structure, which is expected as a consequence of a companion/planet perturbation on or gravitational instability in a young stellar disk. Such a structure has indeed been imaged by ALMA (e.g., in Elias 2-27, Pérez et al. 2016). 


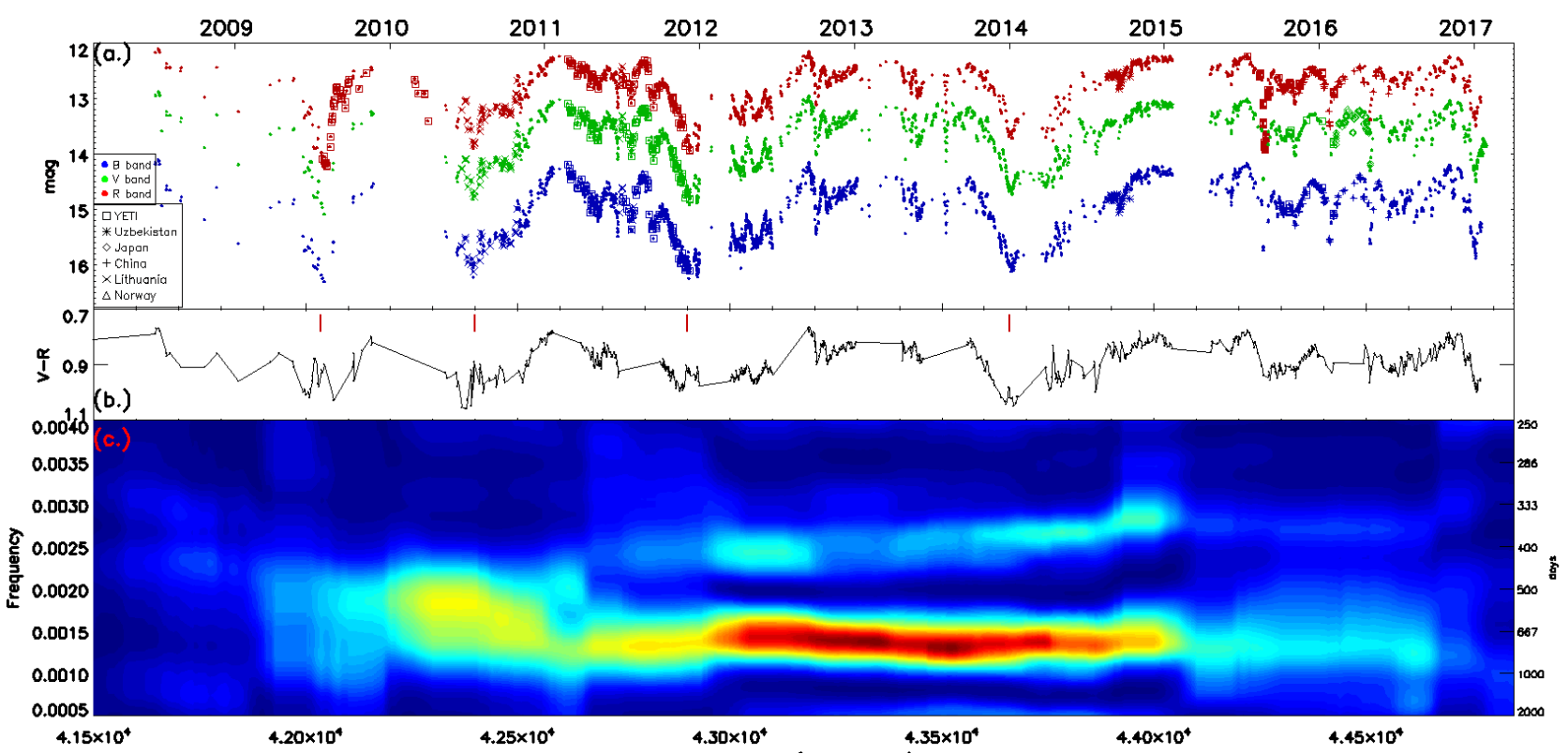

Figure 1: (a) BVR light curves and (b) (V-R) color curve of GM Cep. Note the sudden blueing bumps around many brightness minima marked with red ticks. (c) Dynamical period search with a window size of 2000 days.

\section{Polarization behavior}

A clump (or a string of clumps) in a nearly edge-on orbital plane would modulate the total brightness and polarization in the following way. (1) Near conjunction, with the clump backscattering the starlight, the system is the brightest but with a moderate level of polarization; (2) The polarization becomes the strongest at either quadrature in compliance with the Rayleigh sky model, when the brightness is moderate; (3) Amid the inferior conjunction, the total brightness drops because of the dust extinction, and is moderately polarized due to forward scattering; (4) At the very moment of the minimum, the extinction may become sufficiently high along the line of sight to the star, so that the emergent flux consists predominantly of scattered (blue) light surrounding the sightline.

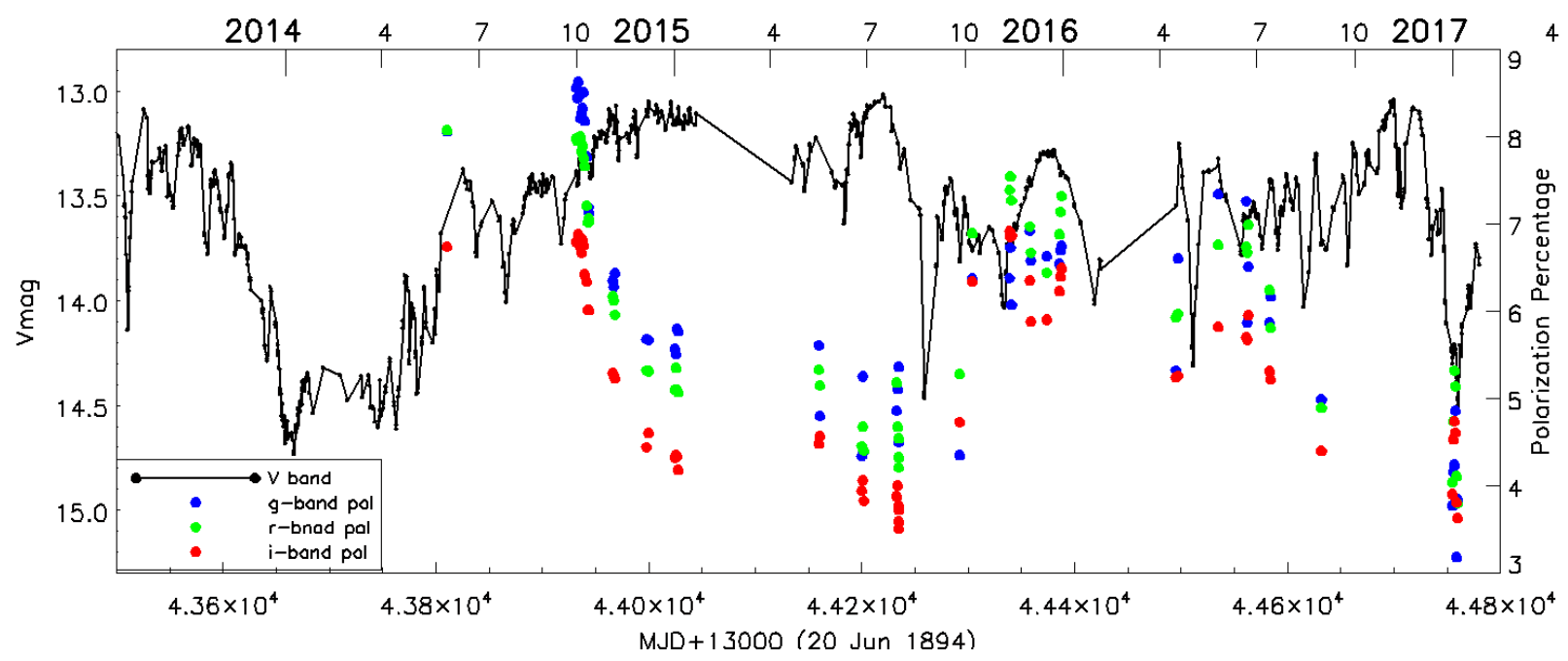

Figure 2: The light curve in V (left axis) versus polarization in the g', r', and i'-bands (right axis) for GM Cep. 
UXors are generally polarized, with the fractional polarization anti-correlated with brightness, reaching maximal polarization during a dip (Grinin et al. 1991; Grinin et al. 1995; Rostopchina et al. 1997). Optical polarization of GM Cep has been carried out by TRIPOL (Triple-Range Imaging Polarimeter)(Sato et al. in preparing) mounted on Lulin One-meter Telescope (LOT) in Taiwan, measuring polarization simultaneously in the Sloan filters g' - $(475.4 \mathrm{~nm})$, r'- $(620.4 \mathrm{~nm})$ and i'-bands $(769.8 \mathrm{~nm})$. In each run, polarized and unpolarized standard stars were observed to calibrate the instrument's polarization.

GM Cep showed a similar, but more intricate behavior (see Fig. 2). From October 2014 to January 2015 , for which the photometric and polarimetric data were both relatively well sampled, the polarization decreased as the star evolved to its maximum brightness. During this epoch, the polarization is higher at a shorter wavelength, peaking at g', which is a manifestation of Rayleigh scattering (i.e., by small particles of gas and/or tiny grains). This may correspond to situation near conjunction as described above (see (1) in first paragraph of this section). From October 2015 to January 2016, a similar anti-correlation seemed to exist, albeit with a shorter time scale and smaller ranges in brightness and polarization variations. Here the polarization peaked at r'-band. This wavelength dependence may be attributed to large scattering particles, such as sub-micron dust grains. It is interesting to note the near the (local) brightness minimum, for which polarization data were available, a comparatively high polarization was detected. Such a phenomenon was also seen in the dip in early 2017, but unfortunately there were no polarization measurements before and after the dip. Follow-up simultaneous photometric and polarimetric monitoring of GM Cep is being carried out.

\section{Summary}

We presented the intensive light curve from mid-2009 to 2017, together with polarization measurements in optical from mid-2014 to 2017. Some major dips ( $>1 \mathrm{mag}$ ) was detected many times, each lasting for a couple of months. The possible existence of a quasi-period with about 500-900 days was found by dynamical period analysis. The anti-correlation between brightness and polarization was discovered by our measurements. Follow-up observations is being carried out.

\section{References}

Briceño C., Hartmann L., Hernández J. et al. 2007, ApJ, 661, 1119

Bouvier J., Grankin K. N., Alencar S. H. P. et al. 2003, A\&A, 409, 169

Chen W. P., Hu S. C. L., Errmann R. et al. 2012, ApJ, 751, 118

Contreras M. E., Sicilia-Aguilar A., Muzerolle J. et al. 2002, AJ, 124, 1585

Goldreich P., Ward W. 1973, ApJ, 183, 1051

Grinin V. P., Kiselev N. N., Minikulov N. KH. et al. 1991, Ap\&SS, 186, 283G

Grinin V. P., Kolotilov E. A., Rostopchina A. 1995, A\&A, 112, 457

Grinin V. P., Rostopchina A. N., Shakhovskoi D. N. 1998, AstL, 24, 802

Herbst W., Herbst D. K., Grossman E. J., Weinstein D. 1994, AJ, 108, 1906

Herbst W., Shevchenko V. S. 1999, AJ, 118, 1043

Hillenbrand L. A. 2008, PhyS, 130, 4024

Johansen A., Oishi J. S., Mac low M. et al. 2007, Nature, 448, 1022

Lomb N. R. 1976, Ap\&SS, 39, 447

Mamajek E. E., Mayer M. R., Hinz P. M. et al. 2004, ApJ, 612, 496

Marschall L. A., Karshner G. B., Comins N. F. 1990, AJ, 99, 1536

Natta A., Testi L., Calvet N. et al. 2007, in Protostars and Planets V (Univ. Arizona Press), 767

Neuhäuser R., Errmann R., Berndt A. et al. 2011, AN, 332, 527

Patel N. A., Goldsmith P. F.,Snell R. L., Hezel T., Xie T. 1995, ApJ, 447, 721

Pérez L M., Carpenter J. M., Andrews S. M. et al. 2016, Sci, 353, 1519 
Rostopchina A. N., Grinin V. P., Okazaki A. et al. 1997, A\&A, 327, 145

Safronov V. S. 1972, Evolution of the protoplanetary cloud and formation of the earth and planets. Jerusalem (Israel): Israel Program for Scientific Translations, Keter Publishing House, $212 \mathrm{p}$

Scargle J. D. 1982, ApJ, 263, 835

Semkov E. H., Peneva S. P. 2012, Ap\&SS, 338, 95

Semkov E. H., Ibryamov S. I., Peneva S. P. et al. 2015, PASA, 32, 11S

Sicilia-Aguilar A., Hartmann L. W., Hernández J., Briceño C., Calvet N. 2005, AJ, 130, 188

Sicilia-Aguilar A., Merín B., Hormuth F. et al. 2008, ApJ, 673, 382

Weidenschilling S. 2000, SSRv, 92, 295

Xiao L., Kroll P., Henden A. 2010, AJ, 139, 1527 